\title{
Validation of the Danish National Diabetes Register
}

This article was published in the following Dove Press journal:

Clinical Epidemiology

18 December 2014

Number of times this article has been viewed

\author{
Anders Green ${ }^{1,2}$ \\ Camilla Sorts $\varnothing^{1,3}$ \\ Peter Bjødstrup Jensen ${ }^{2}$ \\ Martha Emneus' \\ 'Institute of Applied Economics \\ and Health Research, Copenhagen, \\ Denmark; ${ }^{2}$ Odense Patient Data \\ Explorative Network, Odense \\ University Hospital and University of \\ Southern Denmark, ${ }^{3}$ Centre of Health \\ Economics Research, Department of \\ Business and Economics, University of \\ Southern Denmark, Odense, Denmark
}

Correspondence: Anders Green Institute of Applied Economics and Health Research, Ewaldsgade 3, DK-2200 Copenhagen N, Denmark

Tel +45 40887775

Email agreen@dadlnet.dk
Abstract: The Danish National Diabetes Register (NDR) was established in 2006 and builds on data from Danish health registers. We validated the content of NDR, using full information from the Danish National Patient Register and data from the literature. Our study indicates that the completeness in NDR is $\geq 95 \%$ concerning ascertainment from data sources specific for diabetes, ie, prescriptions with antidiabetic drugs and diagnoses of diabetes in the National Patient Register. Since the NDR algorithm ignores diabetes-related hospital contacts terminated before 1990 , the establishment of the date of inclusion is systematically delayed for $\geq 10 \%$ of the registrants in general and for $\geq 30 \%$ of the inclusions before 1997 in particular. This bias is enhanced for ascertainment by chiropody services and by frequent measurements of blood glucose because the date of reimbursement of services, rather than the date of encounter, has been taken as the date of inclusion in NDR. We also find that some $20 \%$ of the registrations in NDR may represent false positive inclusions of persons with frequent measurements of blood glucose without having diabetes. We conclude that NDR is a novel initiative to support research in the epidemiological and public health aspects of diabetes in Denmark, but we also present a list of recommended changes for improving validity, by reducing the impact of current sources of bias and misclassifications.

Keywords: diabetes mellitus, epidemiology, ascertainment, validity

\section{Introduction}

Like the other Nordic countries, Denmark offers unique opportunities for register-based research in epidemiology and health care. ${ }^{1,2}$ Denmark has a particularly strong tradition of epidemiological research in diabetes, ${ }^{3}$ which has been further enhanced with the establishment of the Danish National Diabetes Register (NDR) and the utilization of NDR for research purposes. ${ }^{4,5}$

We have previously used Danish health registers and population-based samples for studies of the epidemiological and public health aspects of diabetes. ${ }^{6,7}$ The establishment of NDR represents a new source for access to individual patient data that we are currently making use of, within the framework of a project called the Diabetes Impact Study 2013, to update our previous studies.

A study that used supplementary methods to ascertain persons with diabetes was performed recently in parts of the former county of Vejle and found that clinical characteristics and mortality differed according to the ascertainment methods applied. ${ }^{8}$ We present here a comprehensive validation analysis of NDR, as part of the Diabetes Impact Study 2013. 


\section{Materials and methods}

\section{Sources and content of NDR}

NDR was established by the Danish National Board of Health in 2006 to provide a resource for monitoring the clinical course in patients with diabetes. ${ }^{4} \mathrm{NDR}$ is built upon information from already existing Danish health registers, including the Danish National Patient Register (DNPaR), ${ }^{9}$ the Danish National Prescription Registry, ${ }^{10}$ and the Danish National Health Service Register ${ }^{11}$ as well as the Danish Civil Registration System (DCRS). ${ }^{12}$ In addition, NDR extracts information from DCRS on residence (municipality code) for each registrant as well as recorded survival status. Linkage of person-specific data between the registers is possible using the person identification number (PIN) that is assigned to each Danish citizen at birth or when granted Danish citizenship. For persons without Danish citizenship but with a permission to stay in Denmark, a temporary PIN is assigned. The PIN is unique and used for administrative purposes throughout the public and private sectors in Denmark, and is saved in the central health registers even after the death of the citizen.

Table 1 provides an overview, based on the account of Carstensen et al, ${ }^{4}$ of the main content of NDR, together with the data sources. The date that a given person is qualified for inclusion in NDR is known for each of the

Table I Source registers and content of NDR

\begin{tabular}{|c|c|c|}
\hline $\begin{array}{l}\text { Source } \\
\text { register }\end{array}$ & $\begin{array}{l}\text { Item in NDR } \\
\text { (label) }\end{array}$ & Inclusion algorithm \\
\hline DNPaR & Ipr (“DMdiag”) & $\begin{array}{l}\text { Registration in DNPaR with a } \\
\text { diagnosis of diabetes, defined as } \\
\text { ICD- I0: DEI0-I4, DH36.0, DO24 } \\
\text { (excluding DO24.4), and ICD-8 (prior } \\
\text { to 1994): } 249,250\end{array}$ \\
\hline \multirow[t]{2}{*}{ DNPrR } & ins (“AntiDiab”) & $\begin{array}{l}\text { Purchase of insulin recorded at least } \\
\text { twice in DNPrR }\end{array}$ \\
\hline & oad (“AntiDiab”) & $\begin{array}{l}\text { Purchase of oad at least twice } \\
\text { recorded in DNPrR. }{ }^{\text {a }} \text { An exception } \\
\text { is females aged } 20-39 \text { prescribed } \\
\text { metformin exclusively }\end{array}$ \\
\hline \multirow[t]{3}{*}{ DNHSR } & blod5il (“BSfreq”) & $\begin{array}{l}\text { Five blood glucose measurements in a } \\
\text { I-year period in DNHSR }\end{array}$ \\
\hline & blod2i5 (“BSfreq") & $\begin{array}{l}\text { Two blood glucose measurements per } \\
\text { year in } 5 \text { consecutive years in DNHSR }\end{array}$ \\
\hline & fodt (“Chiro”) & $\begin{array}{l}\text { Registration of chiropody (as a service } \\
\text { for a diabetic patient) in DNHSR }\end{array}$ \\
\hline
\end{tabular}

Note: anclusion criteria only fulfilled at purchase of second prescription of the drug. Abbreviations: AntiDiab, antidiabetic drug; BSfreq, frequency of blood sugar measurement; Chiro, chiropody; DMdiag, diagnoses of diabetes; DNHSR, Danish National Health Service Register; DNPaR, Danish National Patient Register; DNPrR, Danish National Prescription Registry; ICD, International Classification of Diseases; ins, insulin; NDR, National Diabetes Register; oad, oral antidiabetic drug. data sources (Table 1). The date of inclusion in NDR for a given person occurs when the first of any qualifying events (Table 1) for that person is fulfilled.

It should be noted that according to the detailed documentation, NDR ignores all hospital contacts terminated before 1990 in the search for diabetes-related activities in the DNPaR. ${ }^{13}$ Overall, the algorithms employed are assumed to identify persons with diabetes with a high level of probability and to reduce false positive inclusion of young women with polycystic ovarian syndrome and women with gestational diabetes. NDR is supposed to include all persons with inclusion date since the start of 1997, as well as all persons who were alive at the end of 1996 and identified in the health registers with an inclusion date before 1997.

NDR is operated by the Statens Serum Institut and is updated on an annual basis, most recently by the inclusion of data for the year 2012. The content of NDR is available for research, provided permission is obtained from the Danish Data Protection Agency and the Danish National Board of Health.

\section{Supplementary data}

The present analysis is based on the content of NDR, as extracted on July 3, 2013, containing the data on all inclusions in NDR until 2012. For all registered persons $(n=497,232)$, data were extracted on all contacts (regardless of date) registered in DNPaR, containing information on all interventions performed, primary and secondary diagnoses underlying the contact, hospitalization, and all relevant dates. ${ }^{9}$ The supplementary material presents a list of all diagnoses (according to International Classification of Disease [ICD]8 and ICD10 classifications) and intervention codes (according to the official Danish classification of codes in healthcare) used for this extraction. It should be noted that the codes for diabetes related to pregnancy (ICD10 code DO24) were not used for the purpose of obtaining supplementary data. In addition, all available data on moves, migrations, and deaths recorded on the population of NDR registrants were obtained from DCRS. ${ }^{12}$

\section{Methods of analysis}

All inclusions in NDR were described by ascertainment source. For this purpose, ascertainment by means of a diagnosis of diabetes in DNPaR was labeled "DMdiag". Ascertainment by frequency of blood glucose measurements ("blod2i5" and "blod5i1", respectively) was grouped to the ascertainment category "BSfreq" (Table 1). Similarly, ascertainment by means of purchased antidiabetic drugs 
("ins" and "oad") was grouped to the ascertainment category "AntiDiab". Ascertainment by receiving a service as a person with diabetes by a registered chiropodist was categorized as "Chiro".

Using the access to the full information from DNPaR, we identified for each registrant in NDR, all hospital contacts where diabetes formed part of the primary diagnosis or where interventions performed during the contact were specific for diabetes. The supplementary material contains the list of diagnostic codes (ICD8 classification for contacts before 1995; ICD10 classification for contacts for 2005 and forward) and the procedure codes specific for intervention in diabetes as used in all Danish hospitals. If such contacts were later than the inclusion date registered in NDR, the inclusion date in NDR was set operationally as the date of diagnosis of diabetes. If such contacts were earlier than the inclusion date registered in NDR, the earliest admission date was set operationally as the date of diagnosis.

Secondary ascertainment for a given patient occurred by the fulfillment of inclusion criteria by any other data sources than the first one. For each ascertainment, source methods for survival analysis ${ }^{14}$ were used to estimate the cumulative probability of being ascertained by the first occurring secondary source. Patients were censored if death occurred before experiencing secondary ascertainment.

All data were analyzed using anonymized PINs. Since this was a purely descriptive study without any prior specified hypotheses to be tested, only descriptive analyses were performed.

\section{Ethical aspects}

The Diabetes Impact Study 2013 is observational, with no contact to registered persons. Permission to extract and analyze data was obtained from the Danish Data Protection Agency (permit number Jnr 2013-21-1749) and the Danish National Board of Health (permit number FSEID00000440).

\section{Results \\ Ascertainment by source in NDR}

Figure 1 provides an overview of the population of persons registered in NDR. In total, 688 persons $(0.1 \%)$ have been excluded for various reasons. In particular, 530 persons with inclusion after death represent persons who died

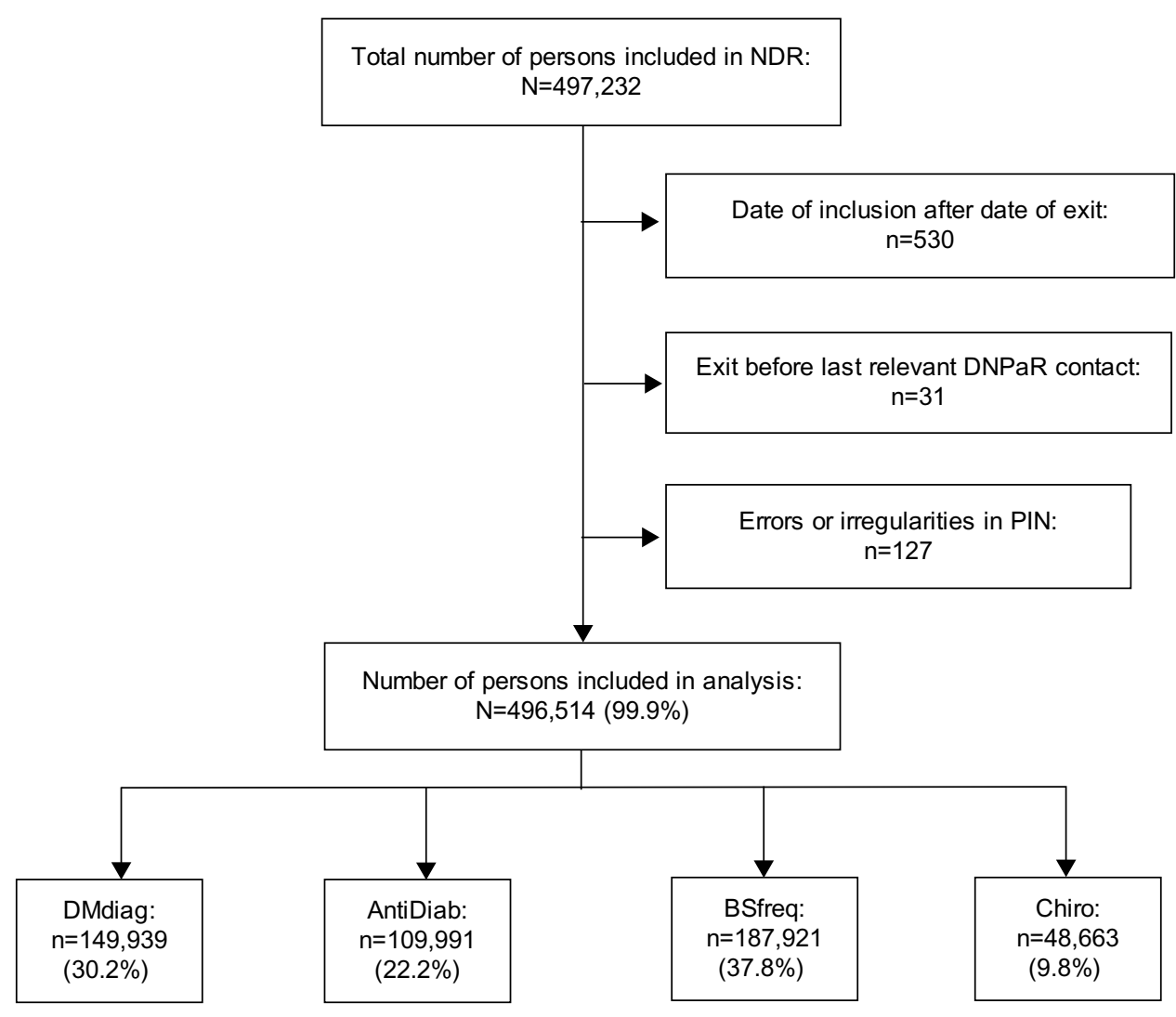

Figure I Flowchart of the population of persons registered as of July 3, 2013 in NDR.

Abbreviations: AntiDiab, antidiabetic drug; BSfreq, frequency of blood sugar measurement; Chiro, chiropody; DMdiag, diagnoses of diabetes; DNPaR, Danish National Patient Register; NDR, National Diabetes Register; PIN, personal identification number. 
before inclusion. Further, 31 persons registered with a relevant contact in DNPaR after exit represent persons with date of emigration registered in DCRS before the event registered in DNPaR. Almost $40 \%$ of registrants are ascertained by "BSfreq" as the primary source, whereas less than $10 \%$ of the registrants are ascertained by "Chiro" as the primary source.

Trends over time in inclusion by primary ascertainment source are illustrated from 1997 and onward in Figure 2. The annual number of inclusions, representing registration incidence, has increased steadily, from about 15,000 to more than 30,000, over the period 1997 through 2011. A relatively large increase has been seen for primary ascertainment by "AntiDiab", while the contribution from "DMdiag" has been rather constant and that for "BSfreq" has been modestly increasing. The number of inclusions by "Chiro" as the primary source is limited but shows relative increases during 2002-2004 and again for the year 2011.

\section{Secondary ascertainment in NDR}

Inclusion in NDR may be qualified in several ways, and registrants may have been ascertained subsequently by other sources than by the primary ascertainment source with the earliest qualification fulfilled. Figure 3 shows the cumulative probability of ascertainment by at least one additional source, by follow-up time from the date of primary ascertainment. This analysis uses inclusions from 1997 throughout 2011. The patterns of cumulative probability vary by primary ascertainment source. For primary ascertainment by both
"DMdiag" and "AntiDiab", some $80 \%$ of the persons will be ascertained by at least one additional source within a period of 10 years, however, with a sharper initial rise for persons primarily ascertained by "DMdiag". Primary ascertainment by "Chiro" is associated with a cumulative probability at a lower level and with a more slow increase. For persons primarily ascertained by "BSfreq", only about $50 \%$ will be ascertained by a second source and with only a modest initial sharp increase in the probability curve.

\section{Operationally defined date of diagnosis of diabetes}

Correspondence between the date of inclusion in NDR and the operationally defined date of diagnosis of diabetes, when using the full historical information in DNPaR, is shown in Table 2, grouped by period of inclusion in NDR. Overall, the operational date of diagnosis equals the date of inclusion in NDR for almost $89 \%$ of persons. However, the percentage with agreement is only about $68 \%$ for persons with inclusion before 1997 against about $98 \%$ for persons with inclusion from 1997 and onward. The proportion of persons with more than 10 years of difference from operationally defined date of diagnosis until inclusion in NDR is about 10\% for inclusion before 1997 against $0.4 \%$ for inclusion from 1997 and onward.

\section{Discussion}

NDR is a nationwide register that provides a unique platform for comprehensive research in the epidemiology and public health aspects of diabetes. As a main advantage, NDR makes

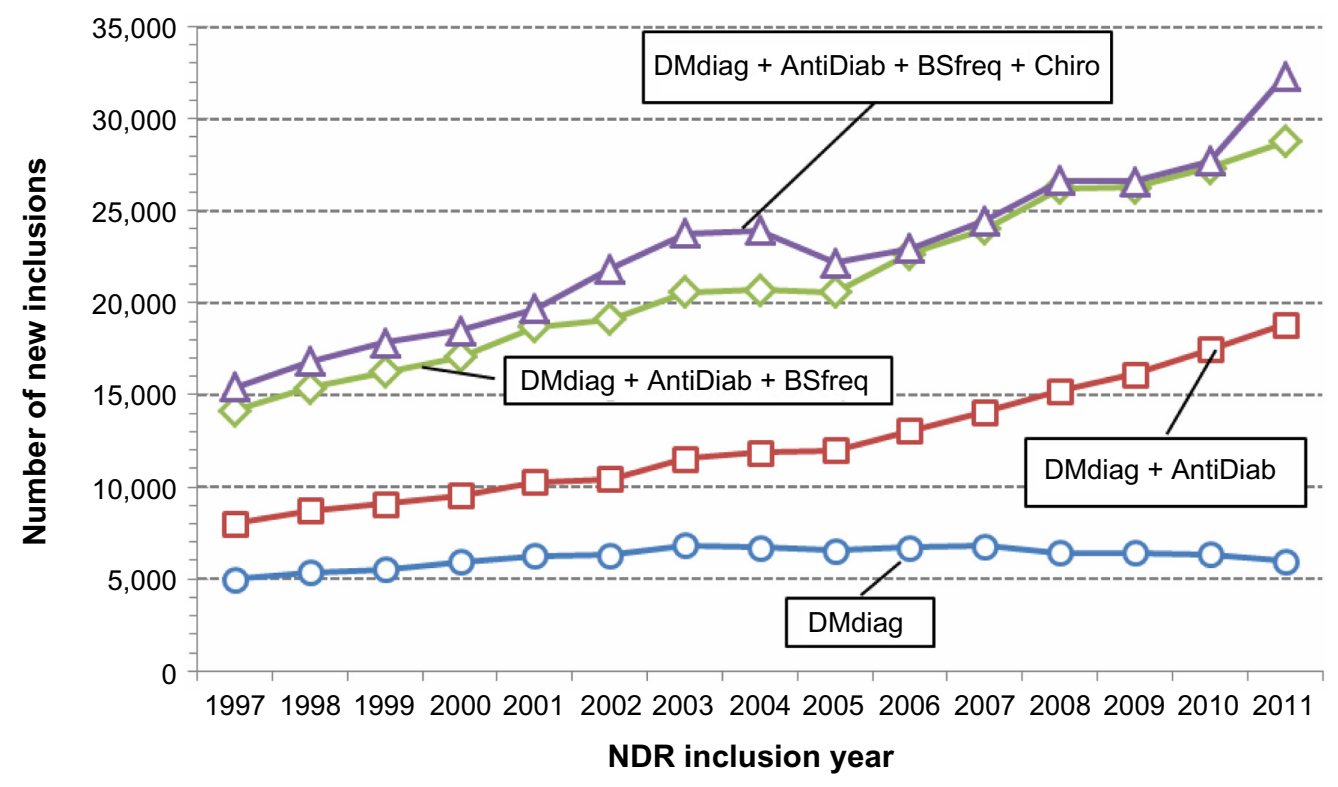

Figure 2 Ascertainment in NDR by source and calendar time, for inclusions in NDR from 1997 through 201 I.

Abbreviations: AntiDiab, antidiabetic drug; BSfreq, frequency of blood sugar measurement; Chiro, chiropody; DMdiag, diagnoses of diabetes; NDR, National Diabetes Register. 


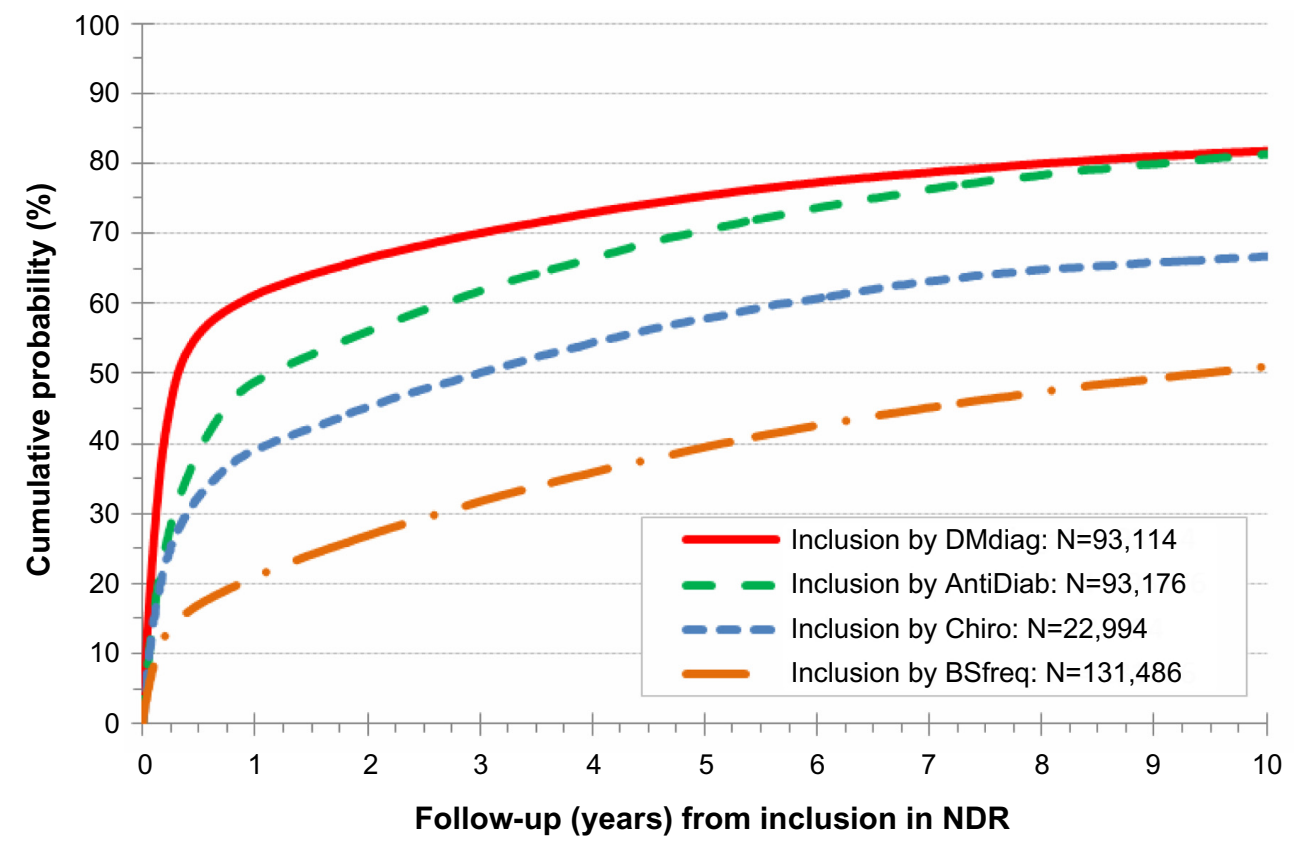

Figure 3 Secondary ascertainment in NDR by primary ascertainment source, for inclusions in NDR from 1997 through 201 I.

Abbreviations: AntiDiab, antidiabetic drug; BSfreq, frequency of blood sugar measurement; Chiro, chiropody; DMdiag, diagnoses of diabetes; NDR, National Diabetes Register.

use of already existing data that are routinely collected and maintained at the national level. As a further advantage, NDR attempts to cover both the secondary and the primary health care sector. In the attempt to validate important elements of NDR, the present study has identified several areas of concern about the validity of NDR, some of which may be alleviated by simple measures.

\section{Validity of ascertainment in NDR}

Ascertainment by purchase of antidiabetic drugs and by a primary hospital discharge diagnosis of diabetes as well as by diabetes-related chiropody may be characterized as specific for diabetes. However, the present list of diagnoses used for the identification of diabetes-related contacts in $\mathrm{DNPaR}$ is not complete and should be supplemented with diagnostic codes for diabetic eye complications. Furthermore, additional relevant contacts may be identified by codes of interventions specific for treatment of diabetes not included in the present NDR algorithm. Such codes may be identified by comparing the codes specified in Table 1 with the codes listed in the supplementary material. The use of such supplementary codes may not increase the ascertainment in NDR substantially but may optimize the establishment of date of inclusion in NDR.

The inclusion of a substantial proportion of persons by means of the frequency of blood sugar measurements in the primary health care sector gives rise to concern about the validity of ascertainment, particularly because currently it is not possible to access information on the results of blood glucose measurements at the national level. Even though validation performed prior to the establishment of NDR indicated a high predictive value of diabetes for the "BSfreq" criteria currently used by NDR, ${ }^{4}$ increased awareness in diabetes may have changed clinical practice and thereby changed

Table 2 Delay (in years) between date of inclusion in NDR and the operational date of diagnosis

\begin{tabular}{|c|c|c|c|c|c|c|}
\hline \multirow[t]{2}{*}{ Delay (in years) } & \multicolumn{2}{|c|}{ NDR inclusion before 1997} & \multicolumn{2}{|c|}{ NDR inclusion 1997-20 I I (incl) } & \multicolumn{2}{|l|}{ Total } \\
\hline & Number & $\%$ & Number & $\%$ & Number & $\%$ \\
\hline No delay & 106,142 & 68.2 & 335,306 & 98.4 & $44 I, 448$ & 88.9 \\
\hline $0<$ delay $<$ I & 2,551 & 1.6 & 2,489 & 0.7 & 5,040 & 1.0 \\
\hline $\mathrm{I} \leq$ delay $<5$ & 13,860 & 8.9 & $\mathrm{I}, \mathrm{I} 40$ & 0.3 & 15,000 & 3.0 \\
\hline $5 \leq$ delay $<10$ & 17,470 & 11.2 & 383 & 0.1 & 17,853 & 3.6 \\
\hline $10 \leq$ delay & $15,7 \mid 7$ & 10.1 & I,456 & 0.4 & 17,173 & 3.5 \\
\hline All persons & 155,740 & 100.0 & 340,774 & 100.0 & 496,514 & 100.0 \\
\hline
\end{tabular}

Abbreviation: NDR, National Diabetes Register. 
the predictive performance of the "BSfreq" algorithms. Our results show that the segment of registrants ascertained by "BSfreq" has a substantially lower probability, at about $50 \%$, of being ascertained subsequently by a secondary source. This finding suggests that the group of persons ascertained exclusively by "BSfreq", to some extent, contains persons with blood sugar examined without having clinically diagnosed diabetes. According to a "worst-case" scenario, $50 \%$ of those $40 \%$ of registrants that are ascertained by "BSfreq" do not have diabetes; this corresponds to about $20 \%$ of the total population registered in NDR.

In a recent study, a regional algorithm based on information on glycosylated hemoglobin $\left(\mathrm{HbA}_{1 \mathrm{c}}\right)$ measurements as a supplementary ascertainment source for the identification of persons with diabetes was compared with the algorithm used by NDR. ${ }^{8}$ Based on an analysis of ascertainment patterns in about 14,000 persons ascertained jointly by the two algorithms, it was estimated that about $21 \%$ of persons ascertained by means of "BSfreq" in NDR may not have diagnosed diabetes but have their blood glucose examined frequently for other reasons. This estimate is close to the one presented above, which was obtained by a completely different approach. Furthermore, almost 700 persons were identified by the regional algorithm as having at least one elevated measurement of $\mathrm{HbA}_{1 \mathrm{c}}$ diagnostic of diabetes but without being registered with NDR. ${ }^{8}$ Taking these findings as representing ascertainment failure in NDR, a proportionate analysis suggests the estimated completeness of diagnosed diabetes in NDR to be $93 \%-95 \%$. Although a full systematic analysis of the completeness of ascertainment has been difficult because of differences in the definitions of the underlying patient populations, it is possible to perform formal analysis for specific segments. Thus, applying capture-recapture methods of analysis to the data in Table 1 of Nielsen et $\mathrm{al}^{8}$ for ascertainment by "AntiDiab" and "DMdiag", the completeness of ascertainment in NDR is estimated at 95.8\% $(95 \%$ confidence interval [CI] 95.4\%-96.3\%) and 97.8\% (95\% CI $97.4 \%-98.6 \%)$, respectively.

Whereas the completeness of ascertained diagnosed diabetes in NDR may be $95 \%$ or higher, a substantial proportion, possibly amounting to about $20 \%$, of the persons registered with NDR may have been falsely included by means of frequent measurement of blood glucose. In June 2012, the Danish National Board of Health decided to accept an $\mathrm{HbA}_{1 \mathrm{c}}$ level above $6.5 \%$ as a diagnostic criterion for diabetes. If readings of $\mathrm{HbA}_{1 \mathrm{c}}$ measurements will be accessible at the national level in the future, it should be considered to revise the algorithm for inclusion in NDR accordingly.
In terms of a full assessment of the performance of the algorithm of inclusion in NDR, the completeness of ascertainment is interpretable as the sensitivity, which we estimate to be $95 \%$ or higher. If further assumed that some $20 \%$ of the registrants in NDR do not have diabetes, the positive predictive value of ascertainment is about $80 \%$. Applying a sensitivity at $95 \%$ and a positive predictive value at $80 \%$ in a scenario with a total population like the Danish, with some six million inhabitants and an annual number of new inclusions in NDR at 30,000, yields an estimated specificity (ie, the proportion of the total population without diabetes and that not ascertained by NDR) at $99.9 \%$.

\section{Date of inclusion in NDR versus operationally defined date of diagnosis of diabetes}

Since the date of clinical diagnosis of diabetes cannot be recorded in NDR, it is necessary to use the date of inclusion as a proxy. This must be done with due reservation because in most cases, the clinical diagnosis may have been established before inclusion in NDR. ${ }^{4}$ The impact of this form of bias may be reduced by searching for the earliest possible registration of diabetes-related contacts in the source registers, notably DNPaR. As per the current NDR algorithm, all hospital contacts closed before 1990 are ignored. ${ }^{13}$ The reasons for this are not obvious, but the ignorance will enhance bias when using the date of inclusion in NDR as a proxy for the date of diagnosis of diabetes. Since our study takes account of all hospital activities, regardless of time, we can show that by ignoring contacts in $\mathrm{DNPaR}$ before 1990 , more than $10 \%$ of the registrants have a delay in the operationally defined date of diagnosis. The delay affects more than $30 \%$ of the registrants with inclusion date before 1997 and is substantial, representing 10 years or even more for about $10 \%$ of the persons included in NDR before 1997. While this may not affect the completeness of ascertainment in NDR, it introduces a bias, particularly in investigations that involve the study of diabetes duration and the impact of diabetes duration over time.

Our study also has identified that persons may be included in NDR after death. To explore on this finding, we investigated further the timing of inclusion in NDR. It appears that for patients primarily ascertained by "DMdiag" and by "AntiDiab", the inclusion dates seem distributed by random, albeit with relatively fewer, inclusions during weekends. In contrast, primary ascertainment by "Chiro" is systematically associated with inclusion on Wednesdays, whereas primary ascertainment by "BSfreq" is associated 
with inclusion also on Wednesday, however with possible spacing between Wednesdays in intervals of up to 4 weeks or even more. Thus, it is likely that for inclusion by methods using contacts with health care providers in the primary health care sector, the inclusion date in NDR represents the date of reimbursement from the National Health Service to the health care provider, rather than the date of actual provision of service. Accordingly, a limited number of patients have died in the interval between the date of contact and the date of reimbursement. This element of the NDR algorithm enhances the bias when using date of inclusion in NDR as a proxy for date of diagnosis.

The present study has demonstrated how administrative changes may influence inclusion in NDR. The number of inclusions by "Chiro" as the primary source is limited but shows a distinct peak for the period 2002-2004 and again for the year 2011. These peaks coincide with periods during which agreements between the chiropodists and the National Health Service were in effect concerning reimbursement of expenses of chiropody for diabetic patients. In fact, the most recent summary report from $\mathrm{NDR}^{15}$ shows a relative decrease in the number of new inclusions for the year 2012, which may be interpreted as a compensation for the relatively steep increase in 2011. Such fluctuations must be interpreted with appropriate caution to the extent that the inclusion rate in NDR is used as a proxy for the incidence rate of diabetes.

\section{Conclusion and recommendations}

NDR represents a novel initiative by which unique opportunities have been created for the monitoring of the basic epidemiological characteristics of diabetes as well as for further in-depth studies on the epidemiological and public health aspects of diabetes in Denmark. Comparisons with other samples of diabetic patients have suggested that NDR may perform with a high level of completeness of ascertainment. ${ }^{8}$ Even though, our study has identified a range of issues that may question other aspects of the validity of NDR, with corresponding consequences for the use of NDR for research purposes. Some of these issues may be resolved by relatively simple changes in the NDR algorithm. Specifically, we recommend the following: 1) In the search for qualifying contacts in DNPaR, the NDR algorithm should include the content of the total DNPaR, rather than being limited to contacts after 1990. This simple measure will reduce substantially the bias in the operationally defined start of the diabetes course that preferentially exists for persons with inclusion date before 1997. 2) The set of diagnostic codes used in the NDR should be reviewed carefully and updated on a periodic basis with current and new relevant diagnostic codes and intervention codes specific for diabetes, as suggested in the supplementary material of this paper. While it may have limited impact only concerning the number of persons identified, such an improvement facilitates the establishment of a more qualified date of inclusion. 3) For inclusion by means of "BSfreq" and "Chiro", the true date of encounter, if available, in the Danish National Health Service Register should be used, rather than using the date of reimbursement. This will further support the establishment of a more qualified date of inclusion, and at the same time, the strikingly invalid feature of including persons after their death will be avoided. 4) As soon as results of $\mathrm{HbA}_{1 \mathrm{c}}$ measurements may become available as part of a future nationwide Danish laboratory database, the NDR algorithm should switch to rely upon the identification of measurements of elevated $\mathrm{HbA}_{1 \mathrm{c}}$, instead of the frequency of blood glucose measurements. Such a switch will significantly reduce the false inclusion in NDR of persons with frequent blood glucose measurements for other clinical reasons than diabetes control. 5) Until the NDR algorithm has been further improved according to the recommendations presented here, we suggest performing sensitivity analyses with respect to the segment of persons ascertained by "BSfreq", in studies that make use of the content of NDR.

\section{Acknowledgments}

This study has been conducted on behalf of the Danish Diabetes Association by the Institute of Applied Economics and Health Research (ApEHR) and supported by a $\mathrm{PhD}$ program at Centre of Health Economics Research (COHERE). Further support has been received as an unrestricted grant from a consortium of sponsors from the pharmaceutical industry, comprising Astra Zeneca/BMS, Novo Nordisk, Merck, Sanofi Aventis, and Bayer.

\section{Disclosure}

The authors report no conflicts of interest in this work.

\section{References}

1. Furu K, Wettermark B, Andersen M, Martikainen JE, Almarsdottir AB, Sørensen HT. The Nordic countries as a cohort for pharmacoepidemiological research. Basic Clin Pharmacol Toxicol. 2010;106(2): 86-94.

2. Thygesen LC, Daasnes C, Thaulow I, Brønnum-Hansen H. Introduction to Danish (nationwide) registers on health and social issues: structure, access, legislation, and archiving. Scand J Public Health. 2011; 39(Supp1 7):S12-S16.

3. Green A. Epidemiologic and public health aspects of insulin-treated diabetes in Denmark. Diabetes Care. 1985;8 Suppl 1:S77-S81.

4. Carstensen B, Kristensen JK, Marcussen MM, Borch-Johnsen K. The National Diabetes Register. Scand J Public Health. 2011;39(Suppl 7): S58-S61. 
5. Carstensen B, Kristensen JK, Ottosen P, Borch-Johnsen K; Steering Group of the National Diabetes Register. The Danish National Diabetes Register: trends in incidence, prevalence and mortality. Diabetologia. 2008;51(12):2187-2196.

6. Green A, Emneus M, Christiansen T, Björk S, Kristensen JK. The Societal Impact of Diabetes Mellitus and Diabetes Care. Report 2: Type 1 Diabetes in Denmark Year 2001. Odense: University of Southern Denmark; 2006. Available from: http://static.sdu.dk//flexpaper/aspnet/Flex_document. aspx?doc=/mediafiles/Files/Om_SDU/Centre/c_ist_sundoke/Forskningsdokumenter/publications/Working papers/20061Christiansen et al. pdf. Accessed August 12, 2014.

7. Green A, Emneus M, Christiansen T, Björk S, Kristensen JK. The Societal Impact of Diabetes Mellitus and Diabetes Care. Report 3: Type 2 Diabetes in Denmark Year 2001. Odense: University of Southern Denmark; 2006. Available from: http://static.sdu.dk//flexpaper/aspnet/Flex_document. aspx?doc=/mediafiles/Files/Om_SDU/Centre/c_ist_sundoke/Forskningsdokumenter/publications/Working papers/20062Christiansen et al. pdf. Accessed August 12, 2014.

8. Nielsen AA, Christensen H, Lund ED, Christensen C, Brandslund I, Green A. Diabetes mortality differs between registers due to various disease definitions. Dan Med J. 2014;61(5):A4840.
9. Lynge E, Sandegaard JL, Rebolj M. The Danish National Patient Register. Scand J Public Health. 2011;39(Supp1 7):S30-S33.

10. Kildemoes HW, Sørensen HT, Hallas J. The Danish National Prescription Registry. Scand J Public Health. 2011;39(Suppl 7):S38-S41.

11. Andersen JS, Olivarius Nde F, Krasnik A. The Danish National Health Service Register. Scand J Public Health. 2011;39(Suppl 7): S34-S37.

12. Pedersen CB. The Danish Civil Registration System. Scand J Public Health. 2011;39(Supp1 7):S22-S25.

13. Statens Serum Institut. Sådan Dannes Det Nationale Diabetes Register [Methods for establishing the National Diabetes Register]. Copenhagen: Statens Serum Institut. Available from: http://www.ssi.dk/ /media/Indhold/DK - dansk/Sundhedsdata og it/NSF/Registre/Diabetesregisteret/ Sådan dannes Det Nationale Diabetesregister.ashx. Accessed August 12, 2014. Danish.

14. Kaplan EL, Meier P. Nonparametric estimation from incomplete observations. J Am Stat Assoc. 1958;53(282):457-481.

15. Brandt CL, Dalum P, Thomsen TT. "I miss the care even though I know it's just a machine": an explorative study of the relationship between an Internet-based smoking cessation intervention and its participants. Health Informatics J. 2013;19(3):233-243. 


\section{Supplementary material}

Table SI Diagnosis codes and intervention codes used for the identification of diabetes-related hospital contacts

\begin{tabular}{|c|c|c|}
\hline Code & Code category & Code text \\
\hline 24900 & ICD8 - disease classification & Insulin-dependent diabetes mellitus, without complications \\
\hline 24901 & ICD8 - disease classification & Cataract, retinopathy in insulin-dependent diabetes \\
\hline 24902 & ICD8 - disease classification & Diabetic nephropathy, Kimmelstiel-Wilson syndrome, insulin-dependent diabetes \\
\hline 24903 & ICD8 - disease classification & Neuropathy, diabetic polyneuritis, insulin-dependent diabetes \\
\hline 24904 & ICD8 - disease classification & Diabetic angiopathy in extremities, insulin-dependent diabetes \\
\hline 24905 & ICD8 - disease classification & Diabetic gangrene, insulin-dependent diabetes \\
\hline 24906 & ICD8 - disease classification & Diabetic coma without ketonuria, insulin-dependent diabetes \\
\hline 24907 & ICD8 - disease classification & Diabetic coma (incl precoma), insulin-dependent diabetes \\
\hline 24908 & ICD8 - disease classification & Insulin-dependent diabetes mellitus, with complications not otherwise specified \\
\hline 24909 & ICD8 - disease classification & Insulin-dependent diabetes mellitus \\
\hline 25000 & ICD8 - disease classification & Insulin independent diabetes mellitus, without complications \\
\hline 25001 & ICD8 - disease classification & Cataract, retinopathy in insulin independent diabetes \\
\hline 25002 & ICD8 - disease classification & Diabetic nephropathy, Kimmelstiel-Wilson syndrome, insulin independent diabetes \\
\hline 25003 & ICD8 - disease classification & Neuropathy, diabetic polyneuritis, insulin independent diabetes \\
\hline 25004 & ICD8 - disease classification & Diabetic angiopathy in extremities, insulin independent diabetes \\
\hline 25005 & ICD8 - disease classification & Diabetic gangrene, insulin independent diabetes \\
\hline 25006 & ICD8 - disease classification & Diabetic coma without ketonuria, insulin independent diabetes \\
\hline 25007 & ICD8 - disease classification & Diabetic coma (incl precoma), insulin independent diabetes \\
\hline 25008 & ICD8 - disease classification & Insulin independent diabetes mellitus, with complications not otherwise specified \\
\hline 25009 & ICD8 - disease classification & Insulin independent diabetes mellitus \\
\hline $\mathrm{BBHA}$ & National Intervention Classification & Peroral diabetes treatment \\
\hline BBHAO & National Intervention Classification & Treatment with peroral antidiabetic agents \\
\hline $\mathrm{BBHAO0}$ & National Intervention Classification & Treatment with agents stimulating insulin production \\
\hline $\mathrm{BBHAOI}$ & National Intervention Classification & Treatment with glucose-lowering antidiabetic agents \\
\hline BBHFO & National Intervention Classification & Insulin treatment \\
\hline BBHF00 & National Intervention Classification & Insulin treatment, mono- or dual therapy \\
\hline BBHFOI & National Intervention Classification & Insulin treatment, basal and bolus therapy \\
\hline BBHF02 & National Intervention Classification & Insulin pump treatment \\
\hline BBKB & National Intervention Classification & Instruction in injecting insulin \\
\hline BUBE0 & National Intervention Classification & Diabetes diet \\
\hline BUBE00 & National Intervention Classification & Diabetes diet, type I diabetes \\
\hline BUBEOI & National Intervention Classification & Diabetes diet, type 2 diabetes \\
\hline BUFA & National Intervention Classification & Dietary instruction in relation to diabetes \\
\hline BUFA0 & National Intervention Classification & Dietary instruction in relation to type I diabetes \\
\hline BUFA00 & National Intervention Classification & Initial dietary instruction in relation to type I diabetes \\
\hline BUFAOI & National Intervention Classification & Follow-up dietary instruction in relation to type I diabetes \\
\hline BUFAI & National Intervention Classification & Dietary instruction in relation to type 2 diabetes \\
\hline BUFAIO & National Intervention Classification & Initial dietary instruction in relation to type 2 diabetes \\
\hline BUFAII & National Intervention Classification & Follow-up dietary instruction in relation to type 2 diabetes \\
\hline BUFA3 & National Intervention Classification & Dietary instruction in relation to pregnant women with type I diabetes \\
\hline BUFA30 & National Intervention Classification & Initial dietary instruction in relation to pregnant women with type I diabetes \\
\hline BUFA3I & National Intervention Classification & Follow-up dietary instruction in relation to pregnant women with type I diabetes \\
\hline DEIO & ICDIO - disease classification & Type I diabetes \\
\hline DEI00 & ICDIO - disease classification & Type I diabetes with coma \\
\hline DEIOOA & ICDIO - disease classification & Diabetic coma, hyperosmolar in IDDM \\
\hline DEIOOB & ICDIO - disease classification & Diabetic coma in IDDM with ketoacidosis \\
\hline DEIOOC & ICDIO - disease classification & Diabetic coma in IDDM without ketoacidosis \\
\hline DEIOOD & ICDIO - disease classification & Diabetic coma, hyperglycemic in IDDM \\
\hline DEIOOE & ICDIO - disease classification & Diabetic coma, hypoglycemic in IDDM \\
\hline DEIOOF & ICDIO - disease classification & Insulin-dependent diabetes with diabetic coma \\
\hline DEIOI & ICDIO - disease classification & Type I diabetes with ketoacidosis \\
\hline DEI02 & ICDIO - disease classification & Type I diabetes with renal complication \\
\hline DEI03 & ICDIO - disease classification & Type I diabetes with eye complication \\
\hline DEI04 & ICDI0 - disease classification & Type I diabetes with neurological complication \\
\hline
\end{tabular}

(Continued) 
Table SI (Continued)

\begin{tabular}{|c|c|c|}
\hline Code & Code category & Code text \\
\hline DEI05 & ICDIO - disease classification & Type I diabetes with complications in peripheral vascular system \\
\hline DEI05A & ICDI0 - disease classification & Type I diabetes with peripheral angiopathy \\
\hline DEI05B & ICDIO - disease classification & Type I diabetes with foot ulcer \\
\hline DEI05C & ICDI0 - disease classification & Type I diabetes with gangrene \\
\hline DEI05D & ICDIO - disease classification & Type I diabetes with microangiopathy \\
\hline DEI06 & ICDIO - disease classification & Type I diabetes with other complication \\
\hline DEIO7 & ICDI0 - disease classification & Type I diabetes with multiple complications \\
\hline DEI08 & ICDI0 - disease classification & Type I diabetes with complication UNS \\
\hline DEIO9 & ICDI0 - disease classification & Type I diabetes without complications \\
\hline DEI09A & ICDIO - disease classification & Type I diabetes UNS \\
\hline DEII & ICDI0 - disease classification & Type 2 diabetes \\
\hline DEI IO & ICDI0 - disease classification & Type 2 diabetes with coma \\
\hline DEIIOA & ICDI0 - disease classification & Diabetic coma in NIDDM without ketoacidosis \\
\hline DEIIOB & ICDI0 - disease classification & Diabetic coma, hypoglycemic in NIDDM \\
\hline DEIIOC & ICDIO - disease classification & Diabetic coma, hyperosmolar in NIDDM \\
\hline DEIIOD & ICDI0 - disease classification & Diabetic coma, hyperglycemic in NIDDM \\
\hline DEIIOE & ICDI0 - disease classification & Diabetic coma in NIDDM with ketoacidosis \\
\hline DEIII & ICDI0 - disease classification & Type 2 diabetes with ketoacidosis \\
\hline DEII2 & ICDI0 - disease classification & Type 2 diabetes with renal complication \\
\hline DEII3 & ICDI0 - disease classification & Type 2 diabetes with eye complication \\
\hline DEII4 & ICDIO - disease classification & Type 2 diabetes with neurological complication \\
\hline DEII5 & ICDI0 - disease classification & Type 2 diabetes with complications in peripheral vascular system \\
\hline DEII5A & ICDIO - disease classification & Type 2 diabetes with peripheral angiopathy \\
\hline DEII5B & ICDI0 - disease classification & Type 2 diabetes with foot ulcer \\
\hline DEII5C & ICDI0 - disease classification & Type 2 diabetes with gangrene \\
\hline DEII5D & ICDI0 - disease classification & Type 2 diabetes with microangiopathy \\
\hline DEI I6 & ICDIO - disease classification & Type 2 diabetes with other complication \\
\hline DEI I7 & ICDI0 - disease classification & Type 2 diabetes with multiple complications \\
\hline DEII8 & ICDIO - disease classification & Type 2 diabetes with complication UNS \\
\hline DEII9 & ICDI0 - disease classification & Type 2 diabetes without complications \\
\hline DEII9A & ICDI0 - disease classification & Type 2 diabetes UNS \\
\hline DEI 2 & ICDI0 - disease classification & Malnutrition-related diabetes \\
\hline DEI 20 & ICDI0 - disease classification & Malnutrition-related diabetes with coma \\
\hline DEI20A & ICDI0 - disease classification & Diabetic coma, hyperglycemic in malnutrition-related diabetes \\
\hline DEI20B & ICDI0 - disease classification & Diabetic coma, hypoglycemic in malnutrition-related diabetes \\
\hline DEI20C & ICDIO - disease classification & Diabetic coma in malnutrition-related diabetes with ketoacidosis \\
\hline DEI20D & ICDI0 - disease classification & Diabetic coma in malnutrition-related diabetes without ketoacidosis \\
\hline DEI20E & ICDI0 - disease classification & Diabetic coma, hyperosmolar in malnutrition-related diabetes \\
\hline DEI2I & ICDI0 - disease classification & Malnutrition-related diabetes with ketoacidosis \\
\hline DEI 22 & ICDI0 - disease classification & Malnutrition-related diabetes with renal complication \\
\hline DEI23 & ICDIO - disease classification & Malnutrition-related diabetes with eye complication \\
\hline DEI 24 & ICDI0 - disease classification & Malnutrition-related diabetes with neurological complication \\
\hline DEI 25 & ICDI0 - disease classification & Malnutrition-related diabetes with complications in peripheral vascular system \\
\hline DEI25A & ICDIO - disease classification & Malnutrition-related diabetes with peripheral angiopathy \\
\hline DEI $25 B$ & ICDI0 - disease classification & Malnutrition-related diabetes with foot ulcer \\
\hline DEI25C & ICDI0 - disease classification & Malnutrition-related diabetes with gangrene \\
\hline DEI25D & ICDI0 - disease classification & Malnutrition-related diabetes with microangiopathy \\
\hline DEI 26 & ICDI0 - disease classification & Malnutrition-related diabetes with other complication \\
\hline DEI 27 & ICDI0 - disease classification & Malnutrition-related diabetes with multiple complications \\
\hline DEI 28 & ICDIO - disease classification & Malnutrition-related diabetes with complication UNS \\
\hline DEI 29 & ICDI0 - disease classification & Malnutrition-related diabetes without complications \\
\hline DEI3 & ICDIO - disease classification & Other forms of diabetes \\
\hline DEI 30 & ICDI0 - disease classification & Other form of diabetes with coma \\
\hline DEI3I & ICDI0 - disease classification & Other form of diabetes with ketoacidosis \\
\hline DEI32 & ICDI0 - disease classification & Other form of diabetes with renal complication \\
\hline DEI33 & ICDI0 - disease classification & Other form of diabetes with eye complication \\
\hline DEI34 & ICDI0 - disease classification & Other form of diabetes with neurological complication \\
\hline
\end{tabular}


Table SI (Continued)

\begin{tabular}{|c|c|c|}
\hline Code & Code category & Code text \\
\hline DEI35 & ICDIO - disease classification & Other form of diabetes with complications in peripheral vascular system \\
\hline DEI35A & ICDIO - disease classification & Other form of diabetes with peripheral angiopathy \\
\hline DEI35B & ICDIO - disease classification & Other form of diabetes with foot ulcer \\
\hline DEI35C & ICDIO - disease classification & Other form of diabetes with gangrene \\
\hline DEI35D & ICDIO - disease classification & Other form of diabetes with microangiopathy \\
\hline DEI36 & ICDIO - disease classification & Other form of diabetes with other complication \\
\hline DEI 37 & ICDIO - disease classification & Other form of diabetes with multiple complications \\
\hline DEI 38 & ICDIO - disease classification & Other form of diabetes with complication UNS \\
\hline DEI39 & ICDIO - disease classification & Other form of diabetes without complications \\
\hline DEI4 & ICDIO - disease classification & Unspecified diabetes \\
\hline $\mathrm{DE} \mid 40$ & ICDIO - disease classification & Diabetes UNS with coma \\
\hline DEI40A & ICDIO - disease classification & Diabetic coma in malnutrition-related diabetes \\
\hline DEI40B & ICDIO - disease classification & Diabetic coma, hyperglycemic in unspecified diabetes \\
\hline DEI40C & ICDIO - disease classification & Diabetic coma, hyperosmolar in unspecified diabetes \\
\hline DEI40D & ICDIO - disease classification & Diabetic coma, hypoglycemic in unspecified diabetes \\
\hline DEI4I & ICDIO - disease classification & Diabetes UNS with ketoacidosis \\
\hline $\mathrm{DEI} 42$ & ICDIO - disease classification & Diabetes UNS with renal complication \\
\hline DEI43 & ICDIO - disease classification & Diabetes UNS with eye complication \\
\hline DEI44 & ICDIO - disease classification & Diabetes UNS with neurological complication \\
\hline DEI45 & ICDIO - disease classification & Diabetes UNS with complications in peripheral vascular system \\
\hline DEI45A & ICDIO - disease classification & Diabetes UNS with peripheral angiopathy \\
\hline DEI45B & ICDIO - disease classification & Diabetes UNS with foot ulcer \\
\hline DEI45C & ICDIO - disease classification & Diabetes UNS with gangrene \\
\hline DEI45D & ICDIO - disease classification & Diabetes UNS with microangiopathy \\
\hline DEI46 & ICDIO - disease classification & Diabetes UNS with other complication \\
\hline DEI47 & ICDIO - disease classification & Diabetes UNS with multiple complications \\
\hline DEI48 & ICDIO - disease classification & Diabetes UNS with complication UNS \\
\hline DEI49 & ICDIO - disease classification & Diabetes UNS without complications \\
\hline DEI59 & ICDIO - disease classification & Hypoglycemic coma UNS \\
\hline DEI59A & ICDIO - disease classification & Insulin shock \\
\hline DG632 & ICDIO - disease classification & Diabetic polyneuropathy \\
\hline $\mathrm{DH} 280$ & ICDIO - disease classification & Diabetic cataract \\
\hline $\mathrm{DH} 360$ & ICDIO - disease classification & Diabetic retinopathy UNS \\
\hline $\mathrm{DH} 360 \mathrm{~A}$ & ICDIO - disease classification & Simplex retinopathy IDDM \\
\hline $\mathrm{DH} 360 \mathrm{~B}$ & ICDIO - disease classification & Proliferative retinopathy IDDM \\
\hline $\mathrm{DH} 360 \mathrm{C}$ & ICDIO - disease classification & Simplex retinopathy NIDDM \\
\hline $\mathrm{DH} 360 \mathrm{D}$ & ICDIO - disease classification & Proliferative retinopathy NIDDM \\
\hline $\mathrm{DH} 360 \mathrm{E}$ & ICDIO - disease classification & Diabetic maculopathy IDDM \\
\hline $\mathrm{DH} 360 \mathrm{~F}$ & ICDIO - disease classification & Diabetic maculopathy NIDDM \\
\hline $\mathrm{DH} 360 \mathrm{H}$ & ICDIO - disease classification & Simplex diabetic retinopathy \\
\hline $\mathrm{DH} 360 \mathrm{~J}$ & ICDIO - disease classification & Proliferative diabetic retinopathy \\
\hline $\mathrm{DH} 360 \mathrm{~K}$ & ICDIO - disease classification & Diabetic maculopathy \\
\hline DZI35AI & ICDIO - disease classification & Screening for diabetic retinopathy \\
\hline
\end{tabular}

Abbreviations: ICD, International Classification of Disease; IDDM, insulin-dependent diabetes mellitus; NIDDM, non-insulin-dependent diabetes mellitus; UNS, unspecified.

Clinical Epidemiology

\section{Publish your work in this journal}

Clinical Epidemiology is an international, peer-reviewed, open access, online journal focusing on disease and drug epidemiology, identification of risk factors and screening procedures to develop optimal preventative initiatives and programs. Specific topics include: diagnosis, prognosis, treatment, screening, prevention, risk factor modification,

systematic reviews, risk \& safety of medical interventions, epidemiology \& biostatistical methods, and evaluation of guidelines, translational medicine, health policies \& economic evaluations. The manuscript management system is completely online and includes a very quick and fair peer-review system, which is all easy to use. 